\title{
The Association of Surrogate Decision Makers' Anxiety and Depression Levels at Baseline with End-of-Life Patient Care Decisions
}

\author{
Andy W. Chen ${ }^{1}$, James E. Slaven ${ }^{2}$, Alexia M. Torke ${ }^{3-5}$
}

${ }^{1}$ Indiana University (IU) School of Medicine, Indianapolis, IN;

${ }^{2}$ Department of Biostatistics, IU School of Medicine, Indianapolis, IN;

${ }^{3} \mathrm{IU}$ Center for Aging Research, Regenstrief Institute, Inc., Indianapolis, IN;

${ }^{4}$ Division of General Internal Medicine and Geriatrics, IU School of Medicine, Indianapolis, IN;

${ }^{5}$ Daniel F. Evans Center for Spiritual and Religious Values in Healthcare, IU Health, Indianapolis, IN

Rationale: Little is known about how the psychological status of surrogate decision-makers (SDMs) influences their decisions regarding end-of-life patient care. Anecdotal evidence suggests high psychological distress may lead to denial of poor prognosis and continued aggressive treatments. We hypothesized that SDM psychological distress is associated with greater use of life-sustaining treatments and lower hospice use for seriously ill older adults.

Objectives: To determine the relationships between surrogate anxiety and depression levels and surrogates' decisions regarding end-of-life treatments.

Methods: This prospective observational study enrolled patient/surrogate dyads from three Midwestern hospitals in one metropolitan area. Eligible patients were 65 years or older and admitted to the medicine or medical intensive care services. Baseline surveys between hospital days 2 and 10 assessed anxiety (GAD-7) and depression (PHQ-9) levels, and SDM preference for do-not-resuscitate (DNR) status. Chart reviews of the electronic medical record and regional health information exchange six months after enrollment identified the use of aggressive life-sustaining treatments and hospice for patients who died.

Results: There were 278 patient/surrogate dyads. After adjusting for other demographic and illness factors, anxiety (adjusted odds ratio [aOR] 1.09; $95 \% \mathrm{Cl} 1.02,1.16 ; P=.007$ ) and depression (aOR 1.08; $95 \% \mathrm{Cl} 1.02$, 1.15; $P=.011)$ were associated with greater preference for DNR status. Among patients who died $(n=115)$, anxiety (aOR 0.89; 95\% Cl 0.79, 0.99; $P=.039$ ) was associated with less use of aggressive life-saving interventions within the last 30 days, and anxiety (aOR 1.23; 95\% Cl 1.09, 1.39; $P<.001$ ) and depression (aOR 1.14; $95 \% \mathrm{Cl} 1.04,1.25 ; P=.004$ ) were associated with greater hospice use.

Conclusions: Contrary to our hypothesis, heightened SDM psychological comorbidities at baseline are associated with greater DNR preference, as well as less life-sustaining treatment and more hospice use at the end of life. More research is needed to understand the impact of psychological distress on surrogate decision-making. 